ARTÍCULO DE COMUNICACIÓN 



\title{
BREVE RECORRIDO POR LA INVESTIGACIÓN EN LA COMUNICACIÓN PARTICIPATIVA DE LATINOAMÉRICA
}

\section{A BRIEF TOUR OF THE PARTICIPATORY COMMUNICATION RESEARCH IN LATIN AMERICA}

\author{
Jorge H. López Rojas ${ }^{1}$ \\ RESUMEN
}

La comunicación participativa, como práctica social y como objeto de estudio, tiene como aspecto particular el ser dinamizada por grupos sociales con propósitos de movilización de sus integrantes que, de manera organizada, gestionan mejores condiciones de vida, generalmente en contradicción y lucha contra poderes establecidos. Se diferencia de las prácticas informacionales que limitan su acción al clásico esquema transmisivo lineal y unidireccional de emisor, mensaje, receptor (E-M-R).

Pese a las características propias que identifican el enfoque participativo de la comunicación, se observa que éstas no son suficientes para ayudar a precisar intencionalidades académicas y objetivos sociales evidentes en prácticas y análisis investigativos, en donde las acepciones se presentan confusas y hasta contradictorias.

El presente estado de arte realiza un recorrido por diferentes enfoques que componen la comunicación participativa en América Latina desde el primer lustro del siglo XX y precisa algunas de sus particularidades con el propósito de aportar a la construcción conceptual en este campo.

Palabras clave: comunicación participativa, participación popular, comunidad, cambio social, desarrollo alternativo.

\begin{abstract}
Participatory communication as a social practice and as an object of study, has the particular feature being energized by social groups for purposes of mobilization of its members who, in an organized, managed, better living conditions, often in contradiction and struggle against established powers. It differs from informational practices that limit their action to the classic scheme transmissive linear and unidirectional sender, message, receiver (EMR).

Despite the characteristics that identify the participatory approach of communication, it is noted that these are not sufficient to help clarify intentions academic and social goals and practices evidenced investigative analysis where the meanings are confusing and even contradictory.

This state of the art includes a tour of different approaches that make participatory communication in Latin America and some of their special needs in order to provide conceptual construction in this area.
\end{abstract}

Key words: participatory communication, popular participation, community social change, alternative development. 


\section{INTRODUCCIÓN}

Dentro del proceso de la investigación "La comunicación comunitaria y la construcción de comunidad en el barrio Potosí", se ha levantado un Estado del Arte sobre el tema de la comunicación participativa. Estudiar el tema de la comunicación comunitaria parte de reconocer un problema conceptual y práctico, como es la dificultad de precisión conceptual evidenciada en publicaciones, discursos y prácticas de actores sociales e institucionales.

El estudio se limita a analizar los trabajos adelantados en América Latina, en razón a las afinidades históricas y culturales propias de la región y su relación con el objeto de investigación. Los pocos estudios hallados sobre el tema específico de la relación comunicación - comunidad, han propiciado que el tema de la investigación se ampliara hacia la comunicación participativa, dado que existen diferentes nominaciones de prácticas sociales similares que expresan como propósito el trabajar por procesos de interacción, mediada o no, que mejoren la vida cotidiana de pobladores que se movilizan organizadamente para alcanzar condiciones de dignidad; muchos de estos trabajos aluden a lo comunitario como algo dado en la existencia de un grupo social confluyente en territorios concretos o en actuaciones conjuntas, en los que poco se profundiza en su análisis crítico y en su conceptualización.

Entre los enfoques más conocidos se encuentran la Comunicación para el Desarrollo (CD), la Comunicación Alternativa (CA), la Comunicación Popular (CP), la Comunicación para el Cambio Social (CCS) y la Comunicación Comunitaria (CC), todos estos con importantes desarrollos prácticos y de reflexión en escenarios de movilización política, y con posterioridad en el mundo académico, en la región latinoamericana.
Este articulo expone un panorama sobre la investigación de la comunicación participativa con propósitos de cambio social que se ha adelantado en América Latina, identificando autores y documentos representativos que han abordado el tema desde diferentes enfoques, al tiempo que se destacan aproximaciones conceptuales - características de grupos sociales denominadas como comunidades.

\section{METODOLOGÍA}

Este artículo conlleva elementos metodológicos propios de un estado de arte, esto es una investigación documental que se realiza con el objetivo de recuperar $y$ trascender reflexivamente en el conocimiento acumulado sobre un tema de estudio. Conocer trabajos afines sobre un tema particular permite comprender y revisar críticamente el conocimiento desarrollado para que el investigador logre una mayor comprensión y así enfocar de manera más asertiva el trabajo que se ha propuesto y contribuya con nuevo conocimiento al campo disciplinar.

La revisión bibliográfica se focaliza a partir de la definición de criterios de búsqueda definidos previamente por el investigador, sin negarse a la posibilidad de modificación de acuerdo a avances logrados. En este caso se precisaron los siguientes criterios:

a. Pertinencia temática: documentos que presentan balances de la investigación en el tema de comunicación participativa en América Latina integrando enfoques cercanos (CD, CP, CCS, CA, CC). Estos enfoques tienen en común el observar la comunicación como un proceso de intercambio de significados y sentidos, socialmente compartidos, cuyo propósito está relacionado con la gestión social de 
cambios en la vida de personas y grupos sociales que tienen comprometida su realización humana en dignidad.

b. Focalización regional: se delimitó a estudios realizados en el tema de la comunicación participativa en América Latina, realizados desde dentro o fuera de la región. Si bien la comunicación participativa caracteriza a América Latina por su amplia práctica y estudios sobre el tema, estos enfoques se trabajan en otros lugares, especialmente en países que se han denominado del tercer mundo, subdesarrollados o emergentes.

c. Referencialidad: la búsqueda de documentos tuvo como punto de partida a autores ampliamente reconocidos por su trabajo como investigadores de la comunicación participativa sobre América Latina, y en las lecturas sucesivas fueron surgiendo otros investigadores menos conocidos, pero con aportes importantes para el estado del arte en desarrollo. Se priorizaron aquellos autores y documentos que fueron citados frecuente y mutuamente. A partir de este reconocimiento se consideró la relevancia del autor y sus textos en la investigación de la comunicación latinoamericana, de esta manera se fortaleció la confiablidad de la fuente.

La razón de optar por el estado del arte ampliado al contexto de América Latina, se encuentra en las similitudes de su desarrollo histórico y político con los procesos sociales colombianos sucedidos a partir de la finalización de la Segunda Guerra Mundial. En este periodo se agudizaron las políticas intervencionistas estadounidenses sobre la región con la implementación del Plan Marshall. Estos temas los analizan con profundidad Beltrán (2005) y Prieto (2006), en relación con los procesos de comunicación y de organización social y política en la región. d. Temporalidad: el trabajo de revisión bibliográfica se concentra en documentos elaborados a partir de 1985, fecha en que se hace la primera compilación de investigaciones enmarcadas en la comunicación alternativa y cambio social por Máximo Simpson. Sin embargo se reconocen avances en el tema desde los años $50 \mathrm{del}$ siglo pasado cuando la CD es impulsada en la región por Estados Unidos a través del Plan Marshall. Este enfoque según los investigadores en este campo se mantiene vigente (Cadavid, 2006, p.5).

e. Tipo y origen de las fuentes: se trabajó con documentos publicados por editoriales $o$ instituciones reconocidas en el ámbito académico de la comunicación y textos publicados por autores especialistas en el tema o divulgados en sitios web que cumplieraon con criterios de referencialidad e institucionalidad de respaldo (universidades, organismos gubernamentales y no gubernamentales).

\section{RESULTADOS}

Realizado este recorrido por la investigación de la comunicación participativa en Latinoamérica se encuentran diferentes maneras de comprensión y práctica de este campo disciplinar que enfatizan en realidades sociales comunes y particulares de esta región. Así mismo, se han abordado enfoques que con cierta regularidad se presentan entremezclados, sin embargo estos permiten hacer comprensiones distintivas en la multiplicidad de estudios y prácticas de la comunicación participativa consultados, que contribuyen en desarrollos teóricos de este campo disciplinar.

\section{Maneras de entender y practicar la comunicación}

La comunicación es un proceso humano y social de interrelaciones de expresión y 
comprensión que involucran momentos de producción, circulación, intercambio desigual - de significaciones, enmarcados cultural e históricamente, y que pueden ser, o no, mediados por tecnologías. Los múltiples mensajes intercambiados son lo que permiten tejer la socialidad, esto es su manifestación en prácticas sociales y discursivas concretas desde donde también se promueven y forman ideologías de actores sociales con interes diferenciados. (Torrico, 2007).

Se diferencian dos formas ideológicas que comprenden y hacen la comunicación en dos perspectivas: la transmisiva y la participativa.

\section{Comunicación como transmisión}

La comunicación es un concepto polisémico. Proviene del término en latín comunis, que significa común. Este origen etimológico ha hecho que la comunicación se defina como el proceso de poner en común cierta información a través de medios por los cuales se traslada de un actor a otro de manera unidireccional. Vista así la comunicación se entiende como un proceso natural de transmisión de ideas que se exponen en un espacio común, esta concepción se refleja en la realidad social cuando se asume que unos actores, generalmente los propietarios de grandes sistemas mediáticos o líderes de opinión, son los dueños de la verdad y tienen toda la autoridad para trasmitirla a otros que no la conocen (Vidal, 2004).

La comunicación entendida como transmisión ha incidido también en el mundo académico. Fuentes (1999) y Sánchez (2002), afirman que los estudios sobre la comunicación han tenido como centro de atención a los medios de información masiva $y$ al modelo de pensamiento eurocentrado, enfoque que desconoce los procesos participativos de la comunicación que se adelantan y se estudian en otras regiones, una de ellas América Latina.

\section{Comunicación como participación}

Una perspectiva diferente de la comunicación la asocia al acto de "compartir", a la "participación" y al intercambio, no solo de información sino también de significados y sentidos. La participación es entendida de muchas maneras, como lo anota Alejandro (2004) quien la explica desde tres dimensiones: (a) la ético-política que considera a las personas como sujetos protagonistas con capacidad y poder para procurar cambios sociales, (b) la económica es pronunciada desde las instituciones para hacer énfasis en criterios de eficacia y eficiencia en el uso de recursos invertidos para el desarrollo y presenta como problema el desconocimiento de prácticas culturales de los pretendidos beneficiarios, y c) la acepción metodológica que la entiende como una herramienta operativa en procesos de intervención social generalmente ligada a proyectos institucionales, la cual con frecuencia termina utilizando a los pretendidos beneficiarios como evidencia superficial de la acción estatal sin traducirse en mejoras notables en condiciones de vida.

La comunicación entendida como participación cobra una dimensión social y política que propone a los sujetos la exigencia del derecho a participar, a ser interlocutores y a superar la actitud de mera recepción a la que los medios masivos y las actitudes autoritarias y verticales les han acostumbrado. Sujetos que el Modelo de Interlocución propuesto por Calvelo (1998) han de incidir en los contenidos de los mensajes de manera clara, comprensible y útil, al tiempo que son reconocidos como portadores de saber y de cultura, que buscan 
con la comunicación resolver necesidades sociales en conjunto y través de su organización política.

La síntesis de esta diferencia conceptual y práctica la presenta Kaplún (1985), con estas características principales: la transmisiva unidireccional como "dominadora" y la participativa multidireccional como "democrática".

Tabla 1: Elementos diferenciales entre la comunicación unidireccional y la participativa.

\begin{tabular}{|ll|}
\hline Comunicación Dominadora & Comunicación Democrática \\
\hline Monólogo & Diálogo \\
Poder & Comunidad \\
Vertical & Horizontal \\
Unidireccional & Doble Vía \\
Monopolizada & Participativa \\
Centrada en las Minorías & Al servicio de las mayorías \\
\hline
\end{tabular}

Fuente: Tomado de Kaplún (1985: 67).

\section{Enfoques Asociados a la Comunicación Participativa}

Estas prácticas de comunicación tienen como elemento común el asumir procesos intencionados en procura de mejorar condiciones de vida de poblaciones en situación de pobreza o malestar social. Sin embargo se constata en los estudios, que con frecuencia, estas denominaciones no son claras y terminan generando confusión, no solo para los investigadores sino a los mismos interesados en promoverlas.

Esta preocupación la comparte Del Valle al considerar que el aglutinar los enfoques mencionados en la comunicación participativa, es una solución cómoda frente a una problemática teórico-metodológica de mayor complejidad que involucra aspectos teóricos, ideológicos, metodológicos, entre otros (2007).

Con el propósito de aportar en la búsqueda de compresiones comunes en la conceptualización y práctica de la comunicación participativa, se presentan las características de cada uno de los enfoques que la componen, desde sus desarrollos históricos.

\section{Comunicación para alcanzar el desarrollo}

Este enfoque se basa en las posibilidades que ofrece la comunicación para ayudar a poblaciones, que sufren situaciones de pobreza económica, a que mejoren sus condiciones de vida y accedan a los beneficios que genera un estadio social imaginado como es el desarrollo. Los primeros proyectos, en este enfoque, se preocuparon primordialmente, por temas como la alimentación, la salud y la educación.

Las prácticas de $C D$ en América Latina durante el primer lustro del siglo XX fueron influenciadas por el gobierno de Estados Unidos, dentro del Plan Marshall, como instrumento para hacer extensivas las innovaciones que llevarían al desarrollo. Con campañas de comunicación, las agencias de cooperación educaban, acompañaban y verificaban que los proyectos implementados propiciaran 
cambios culturales en los pobladores con lo cual mejorarían sus condiciones de vida. Esto no sucedió como se esperaba en los propósitos planteados previamente en los proyectos. Un proyecto pionero en la CD fue el que se adelantó en Colombia a través de Radio Sutatenza, experiencia que ofrecía a los campesinos medios, educativos, especialmente radiales, que fomentaran el desarrollo rural. Se esperaba que los campesinos aplicaran lo que les enseñaban los medios para actividades de producción agropecuaria, de cuidado en la salud y fomento de la educación.

Los proyectos del Plan Marshall llegaron a Latinoamérica en el contexto de la Guerra Fría y de luchas revolucionarias adelantadas en algunos países de esta región, situación que generó conflictos políticos internos. Se criticaban estos proyectos calificados como intervencionistas, lo cual alimentó la identidad del pensamiento latinoamericano de cuestionamiento a modelos foráneos, uno de ellos el de la comunicación transferida linealmente bajo el esquema E-M-R. Beltrán lo analiza de esta manera:

Objetándolo por mecanicista, autoritario y conservador, varios comunicólogos de la región emprendieron, paulatina pero resuelta y creativamente, el diseño de lineamientos básicos para la construcción de un modelo diferente. $O$ sea, que se pusieron a repensar la naturaleza del fenómeno de la comunicación en función de su realidad económica, social, política y cultural. (2005, p.20).

Sin embargo el criticado modelo unidireccional y efectista implementado en la CD logró algunos éxitos. La población, en su momento, creía buena parte de los mensajes emitidos por los medios masivos y en consecuencia lograban transformar su pensamiento y acción. Esto aún se observa en algunos actores, dependiendo de los contextos y situaciones. (Prieto, 2006).
Los años 70 se han conocido por algunos investigadores como la Década de Fuego del pensamiento y las prácticas de comunicación participativa en América Latina. La dura realidad de una promesa de desarrollo que nunca llegó a la gente, condujo a que se adelantaran propuestas de cambio desde los mismos pobladores, así se mejoraron y aumentaron los procesos organizativos $y$ comunicativos mientras se daban enérgicas reflexiones teóricas criticando el modelo económico y las prácticas de comunicación funcionales a éste. (Beltrán 2005, Prieto 2006).

En esta década también se llevaría a cabo la crítica al modelo de desarrollo centrado en la economía por parte de los pensadores latinoamericanos. Beltrán propuso redimensionarlo como un proceso de cambio sociopolítico participativo que "genere transformaciones sustanciales en la economía, la ecología y la cultura de un país a fin de favorecer el avance moral y material de la mayoría de la población del mismo en condiciones de dignidad, justicia y libertad". (Beltrán, 2005:16).

Un aporte contundente a la crítica de la comunicación extensionista en lo rural la hizo Freire (1973) a finales de los años 60 , al plantear que los técnicos agrícolas enviados por las instituciones no lograban dialogar con los campesinos sino que su actuar era indicativo sobre cómo proceder en determinadas tareas. No se escuchaba a los campesinos ni se les reconocía su saber producto de la experiencia. La extensión terminaba violentándolos por medio de prácticas difusivas de invasión cultural.

Con Freire nacen dos conceptos que han cualificado y caracterizado el discurso comunicacional latinoamericano: diálogo e interlocución. Especialmente el concepto de diálogo es característico en el pensamiento freireano, quién lo define como el "encuentro 
amoroso de los hombres que, mediatizados por el mundo, lo pronuncian, esto es, lo transforman $y$, transformándolo, lo humanizan para la humanización de todos" (Freire, 1973, p.46).

El debate socialy académicolatinoamericano sobre la CD llegó hasta la Organización de las Naciones Unidas para la Educación, la Ciencia y la Cultura (UNESCO), para discutir sobre la formulación de políticas nacionales tendientes a democratizar la comunicación en la lógica del Nuevo Orden Internacional de la Información (NOMIC), proceso que no prosperó porque la mayoría de los gobiernos adhirieron al modelo neoliberal con sus consecuentes propuestas de achicamiento del Estado y la libre e ilimitada favorabilidad para los mercados, dentro de ellos el de la información. El informe de la comisión McBride (1980) consolidó estos planteamientos.

Un diagnóstico crítico a las prácticas de la CD lo presenta Prieto (2006) de la siguiente manera: (a) vigencia del modelo tradicional de comunicación E-M-R, (b) reducción de lo comunicacional a medios que traduce la confianza en el poder de los mensajes para cambiar conductas o para transferir tecnologías, y (c) concepción de lo comunicacional como un instrumento de solución de problemas sociales más amplios y no como un elemento de toda cultura humana.

\section{Comunicación popular}

Este enfoque tiene como categoría particular al pueblo como clase social, esto es un grupo poblacional de condiciones económicas limitadas en tanto no tiene como propiedad los medios de producción para satisfacer sus necesidades con el producto de su trabajo.
En América Latina ha existido una tradición cultural de comunicación ligada a prácticas sociales de resistencia ante situaciones adversas propiciadas o facilitadas por los gobiernos de turno. En palabras de Prieto:

Se resistía a gobiernos autoritarios, a la imposición de miserables condiciones de vida, a la exhibición del lujo y del despilfarro por parte de minorías de la sociedad. Se resistía reclamando condiciones de vida dignas y el ejercicio real de la democracia (2006, p.47).

La CP se ha hermanado con la educación popular, en tanto que adopta el principio de comunicar para educar al pueblo, para ayudarlo a organizarse y afrontar los problemas sociales desde su interior y para exigir soluciones a los Estados. El pueblo educado se hace visible en la sociedad para resistir los embates las políticas gubernamentales, y lo comunicacional es apoyo estratégico para realizar una tarea que se mantiene tan vigente como los problemas sociales que le aquejan. Un ejemplo de esta hermandad son las radios populares, especialmente las de los mineros bolivianos, que surgieron por necesidades concretas de grupos sociales en contextos históricos determinados de tal manera que se convirtieron en las emisoras que amplificaban las palabras del pueblo, con sus términos e idiomas, propiciaban la comunicación en su interior . (Beltrán, 2005).

La historicidad de la CP es analizada por Mata (2011) desde tres trayectos, (a) la reclamación de la voz y la palabra, (b) la expresión del conflicto y la construcción de poder, y (c) el acceso a espacios, técnicas y medios de comunicación. En el primero el pueblo fue concebido como "los sin voz", como individuos alienados e imposibilitados para reconocer su explotación por los capitalistas. Ante el silenciamiento, el movimiento de la Teología de la Liberación acompasó las 
prácticas educativas con la comunicación popular, uno de los inspiradores de estos planteamientos fue Paulo Freire.

La idea de un pueblo sin voz ha sido cuestionada. Es evidente que las personas que conforman el pueblo sí tienen palabra, que se expresa en sus propias formas, en algunos casos incomprensibles para los cánones lingüísticos, o incómoda para los poderes establecidos a los que cuestiona, sin embargo siempre es válida. (Mata, 2011).

La palabra del pueblo se pronuncia en todos los espacios y de todas las formas posibles, también en los tiempos actuales de creciente mediatización de la sociedad, esto se observa en las luchas del pueblo para manifestarse en las calles y hacerse ver ante los medios masivos de comunicación. Cuando logran aparecer en estos escenarios, controlados por el poder establecido, logran interrumpir la voz monologa de los dominadores y confrontarla. Sin embargo, la dimensión mediática de la CP ha sido sometida a discusión por sectores radicales, quienes manifiestan perder su condición identitaria de marginalidad. Tal discusión viene desde los años 80 con el surgimiento de la radio educativa, las televisiones obreras y la producción de cine documental realizada desde el pueblo y para el pueblo. (Mata, 2011).

El segundo trayecto histórico de la $C P$ se relaciona con la dinámica del conflicto social y el poder. El pueblo se organiza para reclamar derechos que los Estados no les proveen y para plantear propuestas hacia la superación de condiciones de vida adversa en su dignidad. De ahí se desprenden dos elementos característicos de la politicidad en la CP: (a) la inserción de actores que históricamente dotan al pueblo de una identidad propia con una vocación hegemónica sobre la sociedad, y (b) organicidad legítima de los sectores populares respecto de un proyecto democrático y popular de transformación social. La dimensión política de la CP fue golpeada por las nefastas consecuencias económicas de la imposición del neoliberalismo en la región en los años 90, situación que incidió en rupturas de la organización social popular y con ello la pérdida de horizontes esperanzadores de proyectos colectivos, que hicieron retroceder hacia comportamientos individualistas, así como el desdibujamiento ideológico de actores progresistas o críticos de la iglesia católica. Por otro lado, el neoliberalismo propició los procesos crecientes de concentración de medios de comunicación (Mata, 2011).

Dado que las problemáticas sociales se mantienen vigentes, continúa la necesidad de buscar referentes de inserciones y organicidades para la articulación de la acción política del pueblo en procura de transformaciones sociales hacia la realización digna de las personas. Para Mata (2011), un redimensionamiento de lo popular debe incluir aspectos como la inherente subjetividad de los sujetos, reflejada en su sensibilidad propia, en las interacciones humanas y la articulación de lo público con lo privado, los cuales no fueron tenidos en cuenta en los planteamientos iniciales de la concepción de lo popular. Lo importante era la clase social y no el individuo.

Un tercer trayecto de la CP lo encuentra Mata (2011), en el acceso y uso de medios técnicos de comunicación. Si la comunicación popular es el espacio de expresión de la palabra del pueblo para su encuentro y para discutir el problema del poder en la sociedad, una táctica necesaria es la producción mediática para hacer presencia en los medios masivos, de esta manera, sin perder el referente identitario local, se puede contribuir en 
luchas por la democracia, por el derecho a la información y la comunicación, tanto en ámbitos políticos como en los jurídicos.

\section{Comunicación Alternativa Comunicación "Otra"}

Esta denominación es una de las más problemáticas en la comunicación participativa. El concepto de alter siempre tiene una contraparte que debe ser identificada y definida, incluso para comprender lo propio. Generalmente lo alternativo tiene contraparte en lo establecido, lo hegemónico o lo estatuido en el poder, de ahí que quede un amplio espectro de posibilidades de elegir qué es lo otro diferente de mí o del que no hago parte.

Elementos identitarios de la CA las encuentra Prieto (2006), en el expresarse como diferente y contraria de la comunicación orientada hacia las masas, también en el asociarse a formas de resistencia y disidencia, a la contrainformación, a la acción comunitaria y a las nuevas sensibilidades. Esta perspectiva asume una tensión entre comunicación dominante y comunicación alternativa.

La CA se ha constituido a partir de la denuncia para la concientización señalando formas de actuar de un sistema transnacional y monopólico de la información y los medios masivos, al tiempo que explícita las condiciones de miseria y desigualdad social que los grandes medios invisibilizan (Matterlart \& Doffman, 1971).

Prieto (2006) considera que lo alternativo lo constituye la circunstancia de realización de la comunicación, puede ser un espacio particular, la institución o agente que lo genera, o el contexto en que se desarrolla, aunque en ocasiones es difícil de definirlo.
Lo alternativo puede verse en diferentes ámbitos o prácticas, en los procesos de elaboración de los mensajes, en los mensajes mismos, en su circulación, en la lectura y uso. En el contexto latinoamericano, lo alternativo busca ampliar la democracia, el reclamo de derechos colectivos y de grupos particulares así como la realización de la vida en dignidad. Llevado a la dimensión comunicacional lo alternativo denota los esfuerzos por ampliar espacios de decisión sobre contenidos y formas de los mensajes, por promover organizaciones no verticales así como ejercicios de lectura crítica tanto de los mensajes dominantes como de los alternativos.

El problema referido a la amplitud de cobertura que la CA ofrece a prácticas de comunicación se puede observar en la obra de Simpson (1986) que recoge definiciones, temas y experiencias relacionados con: (a) la hegemonía de monopolios transnacionales de información, (b) la dependencia latinoamericana, (c) la instrumentalización de la llamada otra comunicación, (d) el papel de los Estados en contextos de lucha ideológica, (e) los procesos de autogeneración y decodificación de mensajes en sectores populares, (f) la marginalidad de los medios alternativos $y$ posibilidades de articulación con medios masivos, así como (g) relaciones entre comunicación y culturas populares.

El trabajo que realiza Simpson (1986), parte de la preocupación por las carencias teóricas, empíricas y contextuales, que sufrían las escuelas de comunicación respecto de las experiencias en CA. Para este autor, la comunicación alternativa incluye lo dialógico, lo popular y lo participativo, y tiene como características: (a) acceso amplio de los sectores sociales a los sistemas de comunicación; (b) propiedad social de los medios; (c) contenidos favorables a la 
transformación social; (d) flujos horizontales y multidireccionales de comunicación; y (e) producción artesanal de mensajes.

Los problemas de debilidad conceptual en la CA, en ocasiones, ha provocado en la práctica resultados diferentes y hasta contrarios a los esperados en los proyectos de transformación social. Cabe aquí la autocrítica de Prieto (2006), al recordar que se buscaban alternativas al tipo de sociedad establecida, pero lo que no se pensaba mucho era cómo hacer alternativa a la comunicación misma. Denunciar para concientizar se expresaba en ocasiones como prácticas de violencia discursiva hacia la gente, se creía que los alternativos tenían una verdad por contar, sin importar cómo, y que la gente debía creerla por el solo hecho de ser pronunciada por otros exteriores del sistema imperante. En el afán de concientizar las masas, la comunicación alternativa terminaba usando las estrategias dominantes que se criticaban.

Según Prieto, el propósito de la CA en los años 70, era:

ampliar la participación de la gente en el acceso a las tecnologías y los medios, era impulsarmensajes diferentes a los dominantes, era lograr más diversidad de fuentes de información, era, en suma, el conjunto de aspiraciones de un movimiento a favor de la democratización de la comunicación en la sociedad en general, en la cultura mediática, en las comunidades, en la educación, en el trabajo (2006, p.62).

En desacuerdo con una visión de lo alternativo como proyecto social, diferente de otros, pero hegemónico en cuanto a oposición, y por tanto totalizador y desconocedor de culturas o maneras de ser diversas, González (2011) apuesta por pensar una vida en "otredad", donde la comunicación "otra" reconoce la existencia de comunidades emergentes, locales, grupales, que en autonomía y en conexión en dinámicas de red, se "auto organizan para enfrentar problemas concretos mediante la creación autónoma de sus propios sistemas de información, de comunicación y organización y, desde luego, sus propios sistemas de conocimiento local" (p.13). Tal crítica se fundamenta en la imposición de una alternatividad contra- hegemónica pero de "pensamiento social completo", donde se cree lograr niveles de "consenso" entre "clases aliadas y adversarias", entre hegemónicos y subalternos dominados, explotados, material y simbólicamente.

\section{Comunicación para el cambio social}

El enfoque de la CCS propone diferenciarse de la CD a la que le critica su predominancia economicista, procedimental, difusiva e institucionalizada, para incorporar la dimensión política y de autogestión por parte del grupo social que dinamiza los procesos comunicacionales. Lo que caracteriza la CCS, es el abordaje del problema del poder, que puede ser construido en las comunidades a partir de decisiones sobre su acontecer y proyecciones de futuros que se gestan con luchas hacia la transformación (Gumucio, 2001). La CCS se entiende como procesos de encuentro dialógico entre actores públicos y privados donde sus integrantes mismos definen lo que quieren hacer, a partir de lo que ya son, y desde ahí la comunicación aporta el trabajo colectivo necesario para lograr los cambios deseados (Gumucio \& Tufte, 2008).

Gumucio es de los pocos autores que desde la comunicación participativa esboza una crítica al concepto de comunidad, el cual considera mitificado con frecuencia como un universo social homogéneo, que en la realidad, si bien puede compartir historias 
y prácticas culturales no es exclusivo de ellas, incluso en ocasiones pueden ser conformadas por estratos sociales e intereses divergentes. (Gumucio, 2001).

Por su parte Barranquero (2009), considera que la CCS se caracteriza por su interés de involucrar a la sociedad en su propio proceso de transformación y para ello promueve el diálogo y la participación. Este autor encuentra riqueza de experiencias y reflexiones comunicacionales pioneras en este enfoque en América Latina, que desde la década de los años 70, en abierta critica al positivismo de la ciencia tradicional y el paradigma de la modernidad occidental, han logrado modificar el pensamiento cerrado de la academia estadounidense y generar cambios en el actuar de las agencias de cooperación. Reconoce así la existencia de una comunicología autónoma latinoamericana.

El aporte de la experiencia latinoamericana a la CCS, según Barranquero (2009) es su esencia emancipatoria, la dinámica acción - reflexión - acción, los modos de proceder horizontales y autogestionarios, las prácticas científicas desde un paradigma no eurocentrado como la relación entre educación y la cultura, la pedagogía radical, o las metodologías participativas.

\section{Comunicación comunitaria}

Las relaciones entre comunicación y comunidad son frecuentes en los discursos académicos y de las prácticas sociales, sin embargo es escasa la rigurosidad en su tratamiento conceptual y teórico. Mata (2009) propone algunas reflexiones en este sentido, al considerar que la comunidad, si bien alude a la existencia de un grupo de individuos que interactúan en su interior delimitado, no lo excluye de establecer relaciones con otras experiencias que las exceden. Otra característica de una comunidad es su interacción fundada en valores, significados, fines y expectativas compartidas. Afirmaciones como estas presentan dificultades en razón a la inherencia del conflicto en las interacciones sociales y al ejercicio de poderes que luchan por sus intereses. Es frecuente que esta autocrítica se evada en muchas experiencias de comunicación comunitaria, la cual no siempre obedece a la imagen de lugar cálido o refugio que ofrece seguridad y tranquilidad (Bauman, 2003).

La comunidad no se da en la sola existencia de un grupo social, por el contrario es una construcción continua e interminable que supera barreras y conflictos como los intereses individuales al tiempo que promueve y fortalece los vínculos entre sus integrantes. Por otro lado la comunidad contemporánea ya no está necesariamente ligada a un territorio físico ni aislado, dadas las condiciones actuales de constante interacción en un mundo globalizado.

Superar la mirada endógena y asumir el conflicto permite a las comunidades enfrentarse a construir vidas cotidianas en mejor situación, para ello es preciso hacerse visible en el mundo, relacionarse con el Estado como parte de él, de ahí que de lo comunitario no se excluye el ejercicio de la ciudadanía y de la movilización para hacer realidad los derechos acordados.

Para Uranga (2009), la CC es un enfoque que puede ser aplicable a toda comunicación, cuando es entendida como lugar de encuentro de seres humanos particulares en sus vidas cotidianas, y como proceso social que no se limita a acontecimientos sino a dinámicas que de sujetos históricos que se construyen en diálogo y alteridad. La CC también aporta en la construcción 
de conocimiento social que constituye alimento político que moviliza la acción colectiva y la decisión política.

Una evidencia de la ambigüedad conceptual sobre comunicación y comunidad se puede observar en el trabajo de Rojas, Pérez y De Zúñiga (2010), quienes analizan datos obtenidos por una encuesta que tiene como universo la población urbana, adulta y, civil no institucional en Colombia. El problema teórico en esta producción académica se halla cuando se proponen ilustrar las complejas relaciones entre comunicación y comunidad a través de preguntas sobre las interacciones entre los encuestados y sus vecinos. La información que solicita la encuesta es limitada al tema político y en particular a temas de opinión pública en relación con los medios tecnológicos y los actores gubernamentales. Entre las ambigüedades observadas se encuentra el asumir: que la comunidad es adulta, urbana y no institucionalizada, que un encuestado hace parte de una comunidad, que la comunidad se expresa mayoritariamente en las conversaciones sobre política. Estos pocos cuestionamientos, de los muchos que pueden aparecer, se observan en el cuestionario que se aplicó a 1.026 personas.

Este estudio no asume la comunicación como un proceso social constante y dinámico en el que interactúan personas de diferentes culturas y en contextos particulares, que no se limita a reflexionar sobre temas políticos sino que su convivencia, cuando se da en solidaridad, posibilita abordar problemas del presente para buscarles salidas inmediatas o futuras, así puede decirse que existe una comunidad, lo cual no se da siempre ni en todo grupo social.

\section{Discusión de resultados}

Al evidenciar la existencia de un importante recorrido de prácticas y reflexiones académicas en la comunicación participativa en América Latina, es necesario reconocer que las construcciones teóricas que contribuyan a distinciones en este enfoque, aún son débiles. Se rescatan algunos trabajos que pretenden hacer distinciones al interior del enfoque de la comunicación participativa en autores como Prieto (2006), Beltrán (2005), Mata (2009 y 2011), Gumucio (2001 y 2008), González (2011) y Simpson (1985). Esta situación, que se da por factores como las difusas fronteras del campo disciplinar o la priorización de la práctica sobre la teoría, pueden ser asumidas con mayor rigurosidad, no solo por intereses académicos sino por la confusión observada en discursos que motivan la acción social. Al confundir términos o conceptos en el lenguaje, sucede lo mismo en el sentido de la acción social de la que se esperan cambios para mejorar la vida de las personas que se encuentran en condiciones de insatisfacción de necesidades humanas, materiales y de socialización. Un lenguaje común es la base del entendimiento y la comprensión de actores que hacen parte de un contexto particular, y en el campo de la comunicación participativa, aún por construir, en razón a factores como lo esporádico de este tipo de trabajos, las distancias entre academia y realidad, y la preponderancia vigente de la comunicación transmisiva.

Aunque hay suficiente información y reflexión en la región que podría ser sistematizada para ayudar a organizar el pensamiento de académicos y gestores sociales con el propósito de construir un lenguaje común respecto de las múltiples posibilidades que ofrece la comunicación participativa y así aportar en consolidaciones teóricas que conduzcan a avances cualitativos y orientaciones críticas de las prácticas comunicativas, que se dan 
en gran cantidad y diversidad en América Latina, las propuestas y apuestas que plantea Mata (2011), no serán acciones si la academia no fortalece su estructuración conceptual y con ello el esclarecimiento de las diferentes maneras de estudiar y hacer la comunicación participativa.

$\mathrm{Si}$ bien se encuentran autores que han avanzado en recoger y organizar los recorridos históricos latinoamericanos en la comunicación participativa en América Latina como Prieto (2006) y Beltrán (2005), la difusión de sus trabajos no es muy amplia, ni en los movimientos sociales ni en la academia. Esto se evidencia en el uso de la comunicación de muchas prácticas que dicen llamarse alternativas, como se evidencia en (Simpson, 1985), y de una academia inclinada hacia el transmisionismo comunicacional. Los enfoques que podrían tener elementos participativos como la comunicación para la ciudadanía, el periodismo público, la paz o la convivencia, no alcanzan a tratar el problema participativo de la comunicación.

Este trabajo cumple su propósito en la medida en que ofrezca elementos para ampliar discusiones sobre las características de la comunicación que se dice participativa, pero que en la realidad, por falta de autocrítica o desconocimiento, pueden caer en prácticas transmisivas. Hay importantes ejercicios autocríticos desde las prácticas, y corresponde a los investigadores aportar en este campo para evidenciar de manera crítica los problemas que existen y las maneras en que se pueden superar, en esto también hay mucho por rescatar, desde el presente y desde la historia.

La concepción de comunidad en la contemporaneidad ha de asumir la existencia de grupos sociales que comparten territorios físicos, o no. Lo propio de una comunidad vendría a ser la posibilidad de estar juntos en integración y compromiso en alteridad, con apuestas comunes que hacen vivir un mejor presente avizorando proyectos de futuro entre diferentes. Una de las potencialidades de la comunicación comunitaria es el valorar proyectos comunes, pero también, reconocer la posibilidad de convivir en ciclos fluctuantes de convivencia y conflicto, de esa manera las dificultades manifiestas no impedirán las acciones y esfuerzos que se encaminan en esta perspectiva.

\section{CONCLUSIONES}

Con este trabajo se evidencia la riqueza reflexiva, teórica y empírica de gestores e investigadores de la comunicación participativa en Latinoamérica la cual es resultado del proceso histórico común en la región, signado por el interés de influencia política estadounidense en aspectos políticos, geoestratégicos, económicos y culturales, donde la comunicación se ha visto de diferentes maneras, sea como vehículo de imposición de innovaciones - como dinámica alternativa para la organización social en procura de cambios hacia el bienestar y la dignidad de las personas.

Se ha logrado identificar académicos, mayoritariamente de la región, que han vivido la práctica comunicacional y simultáneamente la reflexionan desde intereses teóricos y políticos. En estos autores la comunicación participativa tiene importantes fuentes de conocimiento $y$ experiencias que pueden aportan a la caracterización de sus enfoques asociados en perspectiva de construir conceptualizaciones que apunten a la comprensión común de sus diferencias como posibilidades y no como fragmentación. 
Existen otros enfoques relacionados con la comunicación participativa como son la comunicación política, la pública, la ciudadana, para la paz o para la salud que no fueron cubiertos en este estado del arte. $\mathrm{Si}$ bien estos enfoques incluyen elementos de participación, por lo general se reducen a estrategias institucionales que desde sus políticas propician acciones unidireccionales y propagandísticas propias de modelos difusivos, por tanto poco participativas. Sería importante aprovechar los escenarios de cambio de perspectiva de los movimientos sociales de la región que han logrado posicionar gobiernos más progresistas. Un reto importante es que las entidades de gobierno y los mismos gestores de la comunicación participativa la comprendan desde una mirada crítica, lo que requiere de esfuerzos similares a los de la construcción de la democracia y la alteridad.

Se evidencia la escasez de reflexiones sobre la concepción de comunidad y su relación con las prácticas comunicacionales que dicen tener que ver con ella, esto permite avizorar un campo de estudio amplio que ofrezca mayores precisiones en torno a sus intencionalidades dando la posibilidad de crítica hacia enunciaciones de características en grupos sociales que dan por hecho la existencia comunitaria.

Se reconocen elementos diferenciales del enfoque latinoamericano al pensamiento eurocentrado sobre la comunicación, aspecto que empieza a superarse pese a las barreras idiomáticas y diferencia de posturas ideológicas, de hecho hay inicios colaborativos importantes con autores españoles en el ámbito académico, lo que representa oportunidades para adelantar programas de investigación y prácticas de intercambio regional, posicionando pensamientos propios en lógicas diferentes al sistema económico capitalista.
La realidad presente muestra que el sistema capitalista y el paradigma desarrollista no han logrado condiciones de vida digna para la mayoría de los seres humanos y muestra de ello es la región latinoamericana. El apostar por la construcción de comunicaciones comunitarias es una apuesta ética y política valerosa que busca romper la individualidad y fomentar la alteridad. 


\section{REFERENCIAS}

Alejandro, M. (2004). La participación: una actitud de diálogo y confianza. En Alejandro, M y Vidal, J. (Comp.).Comunicación y Educación Popular. (pp. 207-212). La Habana:. Editorial Caminos.

Barranquero, A. (2009). Latinoamérica: la arquitectura participativa de la comunicación para el cambio. Diálogos de la comunicación, 78: 1-14. Recuperado en: http:/www.academia. edu.net.

Bauman, Z. (2003). Comunidad: en busca de seguridad en un mundo hostil. Madrid:. Siglo $\mathrm{XXI}$.

Beltrán, L. (2005, julio). La comunicación para el desarrollo en Latinoamérica: un recuento de medio siglo. III Congreso Panamericano de la Comunicación. Universidad de Buenos Aires. Recuperado de: http://www.infoamerica.org/teoria_textos/Irb_com_desarrollo. pdf

Cadavid, A. (2006). ¿Qué comunicación para cuál desarrollo? Documento presentado al Congreso Mundial de Comunicación para el Desarrollo. Roma. Centro de Competencia en Comunicación para América Latina. Recuperado de http://www.c3fes.net/docs/ comunicaciondesarrollocadavid.pdf

Calvelo, J. (1998). Los modelos de información y de comunicación: el modelo de interlocución: un nuevo paradigma de comunicación. FAO. Recuperado de http://www.fao.org/sd/ SPdirect/CDan0022.htm

Del Valle, C. (2007). Comunicación Participativa: Aproximaciones desde América Latina. Redes.Com (113-130).

Freire, P. (1973). ¿Extensión o comunicación?: la concientización en el mundo rural. (22a ed.). México D.F.: Siglo XXI.

Fuentes, R. (1999). La investigación de la comunicación en América Latina: condiciones y perspectivas para el siglo XXI. En Diálogos de la comunicación (56). Recuperado en: http://www.dialogosfelafacs.net/ediciones-anteriores/

González, J. (2011). Un poco de memoria. De lo alternativo a lo comunitario. (Prólogo). En: Krohling, C., Tufte, T., Vega, J., (Ed). Trazos de otra comunicación en América Latina: Prácticas comunitarias, teorías y demandas sociales. Barranquilla.: Uninorte.

Gumucio-Dagron, A., Tufte, T. (2008) (compiladores): Antología de comunicación para el cambio social: lecturas históricas y contemporáneas. La Paz: Consorcio de Comunicación para el Cambio Social, Inc. 
Gumucio-Dagron, A. (2001): Haciendo olas. Historias de comunicación participativa para el cambio social. Nueva York:. The Rockefeller Foundation.

Kaplún, M. (1985). El comunicador popular. Quito:. Editorial Belén.

Mata, M. (2011). Comunicación popular: continuidades, transformaciones y desafíos. En Oficios Terrestres. (26). Recuperado en: perio.unlp.edu.ar/ojs/index.php/oficiosterrestres/ article/view/982

Mata, M. (2009). Comunicación comunitaria en pos de la palabra y la visibilidad social. En Construyendo comunidades, reflexiones actuales sobre comunicación comunitaria. Buenos Aires:. La Crujía.

Matterlart, A., Doffman, A. (1971). Para leer al Pato Donald: Comunicación de masas y colonialismo. (21 ed.). Buenos Aires:. Siglo XXI.

McBride, S. (1980). Un solo mundo, voces múltiples. Comunicación e información en nuestro tiempo. México D.F. Fondo de Cultura Económica.

Prieto, D. (2006). Construir lo pedagógico en América Latina: medio siglo de experiencias en la comunicación y la educación. En Cortés, C., Rosario, A. (Ed). eLearning Comunicación: El diálogo continúa en el ciberespacio (pp. 19-137). San José: Radio Nederland Training Center - RNTC.

Rojas, H., Pérez, I., De Zuñiga, H. (2010). Comunicación y comunidad. Bogotá: Universidad Externado de Colombia.

Sánchez, E. (2002, noviembre). La investigación latinoamericana de la comunicación y su entorno social: notas para una agenda. En Diálogos de la comunicación. 64: 26-36.

Simpson. M. (comp.). (1986). Comunicación alternativa y cambio social: América Latina. México, D.F.: Premia Editores.

Torrico, E. (2007). Acercamiento a la comunicación como cultura académica y a sus proposiciones teóricas generales. En Punto Cero. 14: 41-48.

Uranga, W. (2009). La comunicación comunitaria: proceso cultural, social y político. En Construyendo comunidades, reflexiones actuales sobre comunicación comunitaria. Buenos Aires: La Crujía.

Vidal, J. (2004). Paradigmas básicos en la comprensión del proceso de comunicación. En Comunicación y educación popular. (pp. 21-30). Alejandro, M y Vidal, J. (Comp.). La Habana: Editorial Caminos. 